\title{
Los informes de laboratorio. Su contribución al desarrollo y evaluación de las competencias transversales
}

Juan A. Llorens-Molina

Grupo IEMA, ICE Universidad Politécnica de Valencia. E-mail: juallom2@qim.upv.es

\begin{abstract}
The laboratory learning activities can constitute a suitable educational framework for generic competencies development such as application and practical thinking, analysis and solving problem, teamwork and leadership, etc. For this reason, a key element to evaluate its acquisition and to define the control points can be the assessment of laboratory work, provided that it goes beyond the only manipulative skills or the influence on theoretical knowledge acquisition. It requires a deep methodological change, both concerning its purpose, organization and design and used assessment tools.

In this context, to incorporate critical reflection activities to laboratory reports can provide a valuable information. The results of analysis and encoding of students' reflections from these activities in a 1st course of basic chemistry in Science and Food Technology studies have been discussed in this communication. From these results, several guidelines for development and assessment of generic competencies by means of laboratory work activities have been proposed.
\end{abstract}

Keywords: generic competencies, laboratory work, assessment, critical thinking

\footnotetext{
Resumen

Las actividades de aprendizaje en el laboratorio pueden constituir un marco idóneo para el desarrollo de competencias transversales tales como “aplicación y pensamiento práctic”, “análisis y resolución de problemas” o "trabajo en equipo y liderazgo", etc. Por este motivo, un elemento clave para evaluar su adquisición y definir los puntos de control puede ser la evaluación del trabajo de laboratorio, siempre y cuando ésta vaya más allá de lo puramente manipulativo o de la influencia en la adquisición de conocimientos teóricos. Ello exige un profundo cambio metodológico, tanto en su finalidad, organización y diseño como en los instrumentos de
} 
Los informes de laboratorio. Su contribución al desarrollo y evaluación de las competencias transversales

evaluación utilizados. En este contexto, la incorporación a los informes de laboratorio de actividades de reflexión crítica puede proporcionar una información valiosa. En esta comunicación se discuten los resultados del análisis y codificación de las reflexiones obtenidas de estas actividades en la asignatura Fundamentos de Química, en un primer curso del Grado de Ciencia y Tecnología de Alimentos. A partir de estos resultados se propone un conjunto de orientaciones para el desarrollo y evaluación de las competencias transversales a través del trabajo de laboratorio.

Palabras clave: competencias transversales, trabajo de laboratorio, evaluación, pensamiento crítico.

\section{Introducción}

1. 1. Competencias transversales: el proyecto de la UPV en contextos de aprendizaje diversos

Entre los objetivos del proyecto de incorporación de las competencias transversales (CTs) a la formación de los egresados de la Universidad Politécnica de Valencia se citan expresamente "incorporar las competencias a la formación de los estudiantes utilizando diferentes vías o estrategias" así como "implementar procesos de evaluación y acreditación de dichas competencias en todos los títulos impartidos en nuestra universidad”.

Es importante considerar que el desarrollo de estas competencias tiene lugar en contextos de aprendizaje diversos. Diversidad que posee una influencia decisiva tanto en el terreno metodológico -naturaleza y desarrollo de las actividades- como desde el punto de vista de su evaluación. En este sentido puede afirmarse que el aprendizaje en entornos experimentales posee un conjunto de características específicas que cabe considerar. Éste es precisamente el primer objetivo de este trabajo: describir un conjunto de rasgos específicos del trabajo de laboratorio (TL) que pueden condicionar y a la vez orientar su contribución al desarrollo de las CTs. Posteriormente, se analizará con mayor detalle la relación entre algunos de los objetivos más característicos del TL y cada una de las dimensiones competenciales definidas en el proyecto de la UPV. Por último, se describirá una innovación consistente en la incorporación a los tradicionales informes de laboratorio de una tarea encaminada a suscitar la reflexión de los estudiantes acerca del ambiente de trabajo en el laboratorio y su experiencia personal en torno al mismo. Del análisis de estas tareas se intentará obtener orientaciones para la introducción y evaluación de las CTs.

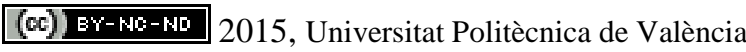

Congreso IN-RED (2015) 


\subsection{El desarrollo de las competencias transversales en el laboratorio: el reto de la complejidad.}

Desde un punto de vista general, puede proponerse la complejidad como rasgo más característico del aprendizaje experimental. Esta afirmación se apoya en los siguientes argumentos:

En primer lugar, en el TL convergen prácticamente todas las categorías definidas en las taxonomías de aprendizaje. En efecto, el conocimiento y comprensión de los hechos y principios científicos se enmarca en la ejecución de tareas donde las habilidades y destrezas psicomotoras poseen un importante papel. Al mismo tiempo el TL requiere el desarrollo de actitudes cuya puesta en práctica es evidente e inmediata: disposición al trabajo colaborativo, sensibilidad medioambiental, cuidado de material e instalaciones de uso común, etc.

En segundo lugar, un aspecto particularmente crítico del trabajo de laboratorio es la función que le otorgamos en los procesos de aprendizaje. Desde la investigación didáctica (Hodson, 1994; Hofstein y Lunetta, 2003; Hofstein, 2004), una crítica generalizada al enfoque habitual de los trabajos de laboratorio es su descontextualización, tanto desde el punto de vista del currículo como en relación al medio socioeconómico y profesional. Una de las principales consecuencias en el terreno metodológico es su ejecución como una secuencia predeterminada de operaciones que deben llevar necesariamente y de modo unívoco al "resultado correcto". La alternativa es evidente: una orientación del TL próxima a la naturaleza del trabajo científico requiere su integración en secuencias de aprendizajes más próximas a estrategias basadas en la resolución de problemas, donde la experimentación es un elemento más de un proceso de indagación que va desde la identificación y análisis de problemas relevantes en la asignatura a la reflexión teórica y a la construcción de nuevos conocimientos. Este enfoque del TL requiere un amplio grado de apertura tanto en el diseño de las actividades (materiales y equipos, métodos de trabajo, etc.) como en su planificación temporal, ya que su realización va estrechamente ligada a la secuencia general de actividades de la asignatura.

Sin embargo, esta orientación choca frontalmente con el esquema organizativo que caracteriza a las sesiones de prácticas en las asignaturas generales de los primeros cursos y particularmente, en el caso de la química, por la dificultad que supone el montaje y la preparación de prácticas diferentes en laboratorios utilizados diariamente por distintos cursos. La pregunta es entonces: ¿Debemos resignarnos a unas prácticas meramente repetitivas, basadas en la simple comprobación de los conocimientos preadquiridos -en el mejor de los casos- y ejecutadas a modo de receta? La respuesta que se pretende aportar en este trabajo es claramente negativa. El marco organizativo en el que nos situamos, aunque

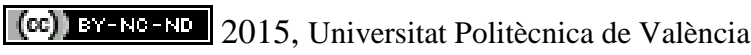

Congreso In-Red (2015) 
Los informes de laboratorio. Su contribución al desarrollo y evaluación de las competencias transversales

manifiestamente mejorable en muchos casos, no deben impedir la incorporación a los TL de elementos que pueden enriquecerlo notablemente. Algunos de estos elementos son:

- La incorporación de actividades previas y posteriores a cada sesión de laboratorio para situarla en el marco de una secuencia coherente desde el punto de vista del contenido y metodológicamente próxima al aprendizaje basado en problemas.

- La progresiva incorporación de un cierto grado de apertura en su diseño en los que los grupos de estudiantes puedan plantearse la aplicación de materiales y procesos alternativos. No es lo mismo, por ejemplo, que todos los grupos determinen el grado de acidez de un vinagre comercial procedente siempre de un mismo envase, a que puedan optar por aplicar ese mismo proceso experimental a diferentes marcas comerciales o incluso diferentes productos: zumos, etc., analizando comparativamente los resultados.

Un tercer aspecto que cabe considerar es de índole más actitudinal. Tal como afirman Hofstein y Mamlok-Naaman (2007), es necesario aproximarse a las diferentes percepciones de los profesores y estudiantes sobre los TL. En efecto, al comienzo de una sesión de laboratorio podríamos formularnos la siguiente pregunta: ¿Qué espero como docente de dicha sesión? Y a continuación trasladar dicha pregunta al punto de vista de los estudiantes. Tradicionalmente, el TL siempre ha suscitado una motivación intrínseca en el alumnado simplemente por el marco en el que se desarrolla, más activo, dinámico y facilitador de la interacción entre los propios estudiantes y con el profesorado. Es evidente que, si como fruto del TL lo único que solicitamos al alumnado es un conjunto de datos experimentales que indican si la práctica "ha salido" bien o no, no vamos a obtener información relevante sobre esa posible divergencia de motivaciones entre docente y discente. Si, por el contrario, solicitamos al estudiante que reflexione sobre los problemas, logros y dificultades que ha experimentado, podemos obtener una valiosa información acerca de cómo reorientar los TL, qué aspectos deben cuidarse más y cuáles son más propicios como punto de partida para el desarrollo de competencias transversales.

Por último, la evaluación de las competencias tanto generales como específicas no puede centrarse exclusivamente en los logros de los estudiantes, al margen de un enfoque sistémico de la evaluación. Es imprescindible conocer la influencia de los distintos factores que inciden en los procesos de aprendizaje para introducir la retroalimentación adecuada. En el trabajo de laboratorio inciden notablemente factores de naturaleza organizativa y de infraestructura que afectan al aprendizaje de los estudiantes y a su propia percepción del mismo. Esta influencia ha sido claramente observada en los resultados de trabajos anteriores (Llorens-Molina et al. 2009, 2012a, 2012b) basados en el análisis de las preguntas que el alumnado formula espontáneamente durante las sesiones de laboratorio (Students’ generated questions -SGQs-) (Pedrosa de Jesús, 2003, 2007). Aspectos tales como la disponibilidad y localización de material y reactivos, discordancias entre la 
información proporcionada por los guiones de prácticas y lo que realmente se utiliza en el laboratorio, el modo de organizar el trabajo con un equipo de uso colectivo, etc., contribuyen notablemente a la existencia de un "ruido" (Byrne, 1990) que resta fluidez y eficacia a la comunicación entre los propios estudiantes y entre estos con el profesorado, imprescindible para un buen desarrollo del TL.

\subsection{El concepto de ambiente de aprendizaje: un marco teórico de interés para la incorporación de las CTs y su evaluación.}

Tras el anterior análisis, es comprensible que desde la investigación didáctica se haya prestado especial atención al concepto de ambiente de aprendizaje (Learning Environment). Éste puede constituir una aproximación conceptual más coherente, válida y fiable para la caracterización y evaluación de las innovaciones didácticas (Wilson, 1995. Brown y Cole, 2000). Tal como lo define Duarte (2009), un ambiente de aprendizaje es "un espacio y un tiempo en movimiento, donde los participantes desarrollan capacidades, competencias, habilidades y valores". Consecuentemente, su caracterización no puede limitarse a la identificación de ciertas características básicas de carácter material (infraestructuras, recursos, etc.) o a la especificación de algunas pautas de interacción entre docentes y estudiantes, sino que debe adentrarse en aspectos ligados al desarrollo humano y afectivo, así como en la relación entre contexto y aprendizaje.

Se han propuesto numerosos instrumentos para caracterizar los ambientes de aprendizaje. En el terreno de las prácticas de laboratorio, cabe citar el SLEI (Science Laboratory Environment Inventory). Este instrumento ha sido aplicado en numerosas investigaciones con muestras muy numerosas de estudiantes (Mc Robbie et al., Fisher et al., 1998; Hofstein et al., 2001). Está constituido por cuestionarios de carácter objetivo y en él se distinguen las siguientes escalas:

- Cohesión entre los estudiantes, es decir, en qué medida comparten conocimientos, ayudan y son ayudados por otros.

- Divergencia de las actividades de laboratorio o medida en que su desarrollo puede considerarse abierto.

- Integración, o grado en que las actividades del laboratorio forman parte de un conjunto coherente y estructurado con el resto de actividades del curso.

- Claridad de las reglas que determinan la organización y desarrollo de las sesiones de laboratorio.

- Aspectos de infraestructura: adecuación de los materiales y equipos.

Dada la naturaleza de estas escalas y el contenido de las pruebas, la adaptación del SLEI puede considerarse una contribución potencialmente valiosa, no solo para la evaluación de las CTs en sí mismas, sino sobre todo para analizar en qué medida el ambiente de aprendizaje en el laboratorio está facilitando u obstaculizando su adquisición.

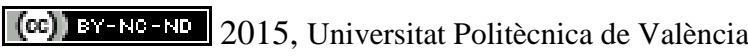

Congreso In-Red (2015) 
Los informes de laboratorio. Su contribución al desarrollo y evaluación de las competencias transversales

\subsection{El trabajo de laboratorio y la adquisición y evaluación de competencias transversales.}

Hasta aquí se han discutido las principales características del trabajo de laboratorio como actividad de aprendizaje y se han descrito las principales dificultades a las que se enfrenta su puesta en práctica en nuestro contexto. También se ha propuesto el concepto de ambiente de aprendizaje como marco teórico en el que situar el desarrollo y evaluación de las CTs relacionadas con el trabajo experimental.

Desde esta fundamentación teórica, partiendo de las dimensiones competenciales descritas en el proyecto de la UPV (Tabla 1), se propone un conjunto de objetivos para el trabajo de laboratorio potencialmente vinculados a la adquisición de dichas competencias. Se sugiere también, en cada caso, un conjunto apropiado de instrumentos de evaluación (Tabla 2).

Tabla 1. Dimensiones competenciales UPV.

\begin{tabular}{|c|c|c|}
\hline 1. & Comprensión e integración & $\begin{array}{l}\text { 2. Aplicación y pensamiento } \\
\text { práctico }\end{array}$ \\
\hline 3. & $\begin{array}{l}\text { Análisis y resolución de } \\
\text { problemas }\end{array}$ & $\begin{array}{l}\text { 4. Innovación, creatividad y } \\
\text { emprendimiento }\end{array}$ \\
\hline 5. & Diseño y proyecto. & $\begin{array}{l}\text { 6. Trabajo en equipo } \mathrm{y} \\
\text { liderazgo. }\end{array}$ \\
\hline 7. & $\begin{array}{lr}\text { Responsabilidad } & \text { ética, } \\
\text { medioambiental } & \mathrm{y} \\
\text { profesional. } & \end{array}$ & Comunicación efectiva. \\
\hline 9. & Pensamiento crítico & $\begin{array}{l}\text { 10. Conocimiento de } \\
\text { problemas contemporáneos }\end{array}$ \\
\hline 11. & Aprendizaje permanente. & $\begin{array}{l}\text { 12. Planificación y gestión del } \\
\text { tiempo. }\end{array}$ \\
\hline
\end{tabular}

Tabla 2. Objetivos del TL, CTs e instrumentos de evaluación

\begin{tabular}{lll}
\hline Objetivos & CTs & Instrumentos de evaluación basados \\
\hline $\begin{array}{l}\text { Reconocer problemas relevantes desde el punto de } \\
\text { vista social o profesional, relacionados con el TL. }\end{array}$ & $\begin{array}{l}\text { Cuestionarios pre-laboratorio, rúbricas } \\
\text { sobre elementos del informe, SLEI. }\end{array}$ \\
$\begin{array}{l}\text { Reconocer el objetivo del TL y el significado de los } \\
\text { resultados que se pretende obtener. }\end{array}$ & $\begin{array}{l}\text { Pruebas objetivas pre-laboratorio, } \\
\text { prueba escrita integrada en la } \\
\text { asignatura, SLEI. }\end{array}$ \\
$\begin{array}{l}\text { Aplicar los conceptos y la terminología adecuada } \\
\text { para formular problemas, describir el proceso de } \\
\text { resolución y discutir los resultados obtenidos. }\end{array}$ & $\begin{array}{l}\text { Rúbricas sobre elementos del informe, } \\
\text { cuestionarios post-laboratorio, prueba } \\
\text { escrita integrada en la asignatura, } \\
\text { presentaciones orales o posters. }\end{array}$
\end{tabular}

(c)) EY-NC-ND 2015, Universitat Politècnica de València

Congreso IN-RED (2015) 
Tabla 2. Continuación

Proponer o analizar críticamente los protocolos experimentales seleccionando los materiales e instrumentos adecuados.

Utilizar correctamente y de modo seguro las instalaciones y equipos del laboratorio, aplicando criterios de seguridad y respeto al medio ambiente.

Reconocer procesos industriales o de la vida profesional directamente relacionados con las operaciones realizadas en el laboratorio.

Elaborar de modo cooperativo los informes de 6,8 laboratorio.

Tomar decisiones de manera autónoma frente a las incidencias y problemas que surgen en el TL.

Utilizar con autonomía y rigor las fuentes de 9 información adecuadas.

Contrastar críticamente los resultados.
2,5

7,13

1,10

$2,3,4,7$

(1)

1,9
Participar mediante propuestas razonadas en la mejor organización y desarrollo de los TL.

Hojas y protocolos de observación, rúbricas sobre elementos del informe.

Protocolos de $\begin{gathered}\text { observación, } \\ \text { cuestionarios de autoevaluación y } \\ \text { coevaluación }\end{gathered}$

Rúbricas sobre elementos del informe, pruebas escritas.

Rúbricas sobre elementos del informe, autoevaluación del trabajo cooperativo, SLEI

Protocolos de observación, registro de preguntas (SGQs)

Rúbricas sobre elementos del informe, presentaciones o posters

Rúbricas sobre elementos del informe, cuestionarios post-laboratorio

4,5,7,8,9 Protocolos de observación, registro de preguntas (SGQs), SLEI, actas de reuniones de planes de acción tutorials

\begin{tabular}{lll}
\hline $\begin{array}{l}\text { Distribuir y coordinar las tareas adecuadamente } \\
\text { dentro del grupo. }\end{array}$ & Protocolos de observación, SLEI \\
$\begin{array}{l}\text { Planificar la ejecución del TL y realizarlo dentro del } \\
\text { tiempo asignado. }\end{array}$ & $\begin{array}{l}\text { Protocolos de observación, rúbricas } \\
\text { sobre elementos del informe. }\end{array}$ \\
$\begin{array}{l}\text { Resumir con brevedad los objetivos globales del TL } \\
\text { y secuenciar correctamente sus operaciones. }\end{array}$ & $\begin{array}{l}\text { Rúbricas sobre elementos del informe, } \\
\text { realización de un diagrama de flujo } \\
\text { previo a la sesión. }\end{array}$
\end{tabular}

\section{Objetivos}

Partiendo de la descripción general propuesta anteriormente, el objetivo de este trabajo se centra en la introducción de actividades de reflexión en los informes de laboratorio de los estudiantes. De un modo más concreto pueden enumerarse los siguientes objetivos específicos:

- Analizar la información proporcionada por la reflexión crítica de los estudiantes expresada a través de los informes del laboratorio mediante la codificación y análisis de la misma.

(c)) EY-NC-ND 2015, Universitat Politècnica de València

Congreso In-Red (2015) 
Los informes de laboratorio. Su contribución al desarrollo y evaluación de las competencias transversales

- Comparar dicha información con la procedente de anteriores investigaciones basadas en el análisis de las preguntas formuladas espontáneamente por los estudiantes durante las sesiones de laboratorio.

- Discutir las consecuencias de los análisis anteriores en el diseño y gestión de las prácticas de laboratorio, incidiendo particularmente en las actividades online asociadas.

- Proponer, a modo de conclusión, un catálogo de buenas prácticas docentes que favorezcan el desarrollo de la autonomía en los estudiantes así como la adquisición de determinadas competencias transversales.

\section{Desarrollo de la innovación}

Se solicitó una actividad de reflexión acerca de las dificultades y los aspectos positivos y negativos más relevantes de cada sesión de prácticas de Química Orgánica, proponiendo también la formulación de sugerencias para su mejora. El enunciado de la actividad, tal como se propuso a los estudiantes es:

Texto de la tarea

1. Describid del proceso experimental, si ha habido alguna incidencia, algún fallo que se ha tenido que corregir, etc.

2. Añadid en cada informe una breve reflexión sobre la práctica:

A. Aspectos positivos. Qué he aprendido. Aspectos que más me han llamado la atención o me han resultado interesantes. Aspectos positivos de la organización, contenido y docencia de las prácticas.

B. Aspectos negativos. Conceptos u operaciones que me han resultado especialmente confusos. Aspectos que no me han generado interés. Aspectos negativos de la organización, contenido y docencia de las prácticas.

C. Dudas sobre los fundamentos teóricos y los procedimientos experimentales de la práctica.

D. Sugerencias para la mejora de las prácticas.

Se procesaron 82 informes (documento primario) correspondientes a tres prácticas de química orgánica correspondientes a la asignatura Fundamentos de Química, en el primer curso del Grado de Ciencia y Tecnología de los Alimentos. Mediante el software Atlas.ti se obtuvieron y codificaron 215 citas. El proceso de codificación abierta permitió el establecimiento de un conjunto de familias de códigos y finalmente un conjunto de supercódigos cuyo significado está relacionado con el ámbito social, académico o personal en el que cabe situar la reflexión, opinión o sugerencia formulada.

(c)) EY-NC-ND 2015, Universitat Politècnica de València

Congreso IN-RED (2015) 


\section{Resultados}

El proceso de codificación descrito conduce a dos redes conceptuales: la primera (figura 1) muestra aquellos aspectos positivos sobre los que determinadas competencias transversales pueden desarrollarse. El otro mapa recoge aquellos aspectos negativos o problemáticos que pueden obstaculizarlas (figura 2).

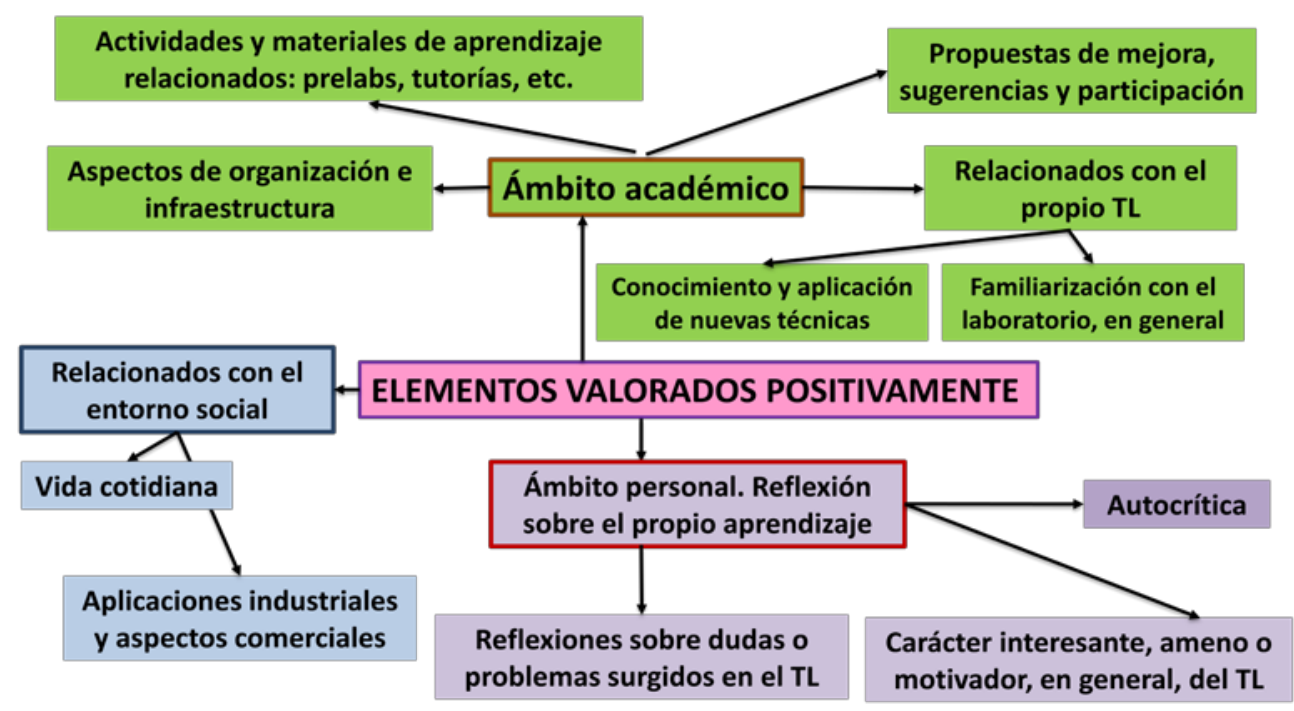

Fig. 1. Elementos del TL valorados positivamente por los estudiantes

Al analizar estos resultados se pueden distinguir tres grandes ámbitos: (1) El académico, relacionado con la organización del trabajo, su contenido y los materiales didácticos; (2) el social, relacionado con aspectos aplicados y de la vida cotidiana y (3), el personal, relacionado con la percepción del TL por el estudiante y la reflexión sobre su trabajo. Su portancia relativa en los informes procesados queda reflejada en el diagrama de la figura 2.

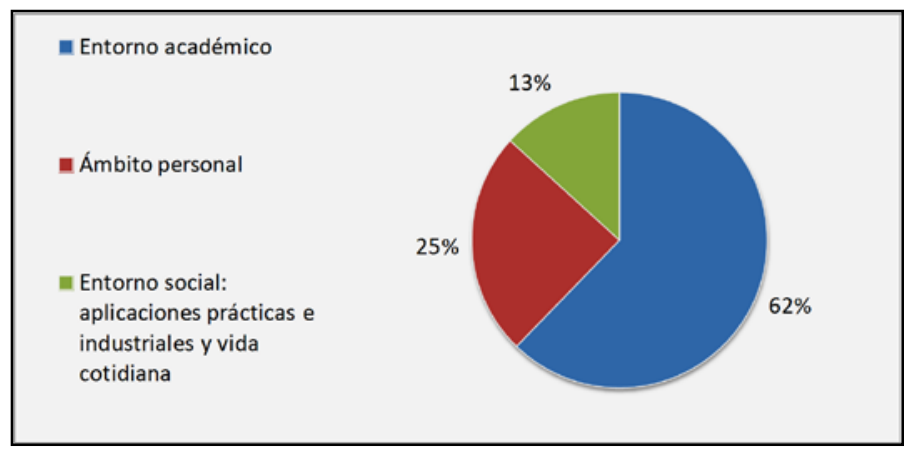

Fig. 2. Importancia relativa de los ámbitos académico, social y personal

(c)) EY-NC-ND 2015, Universitat Politècnica de València

Congreso In-Red (2015) 
Los informes de laboratorio. Su contribución al desarrollo y evaluación de las competencias transversales

De un modo más detallado, los diagramas de las figuras 3 y 4 se muestran los aspectos predominantes dentro de los ámbitos académico y personal.

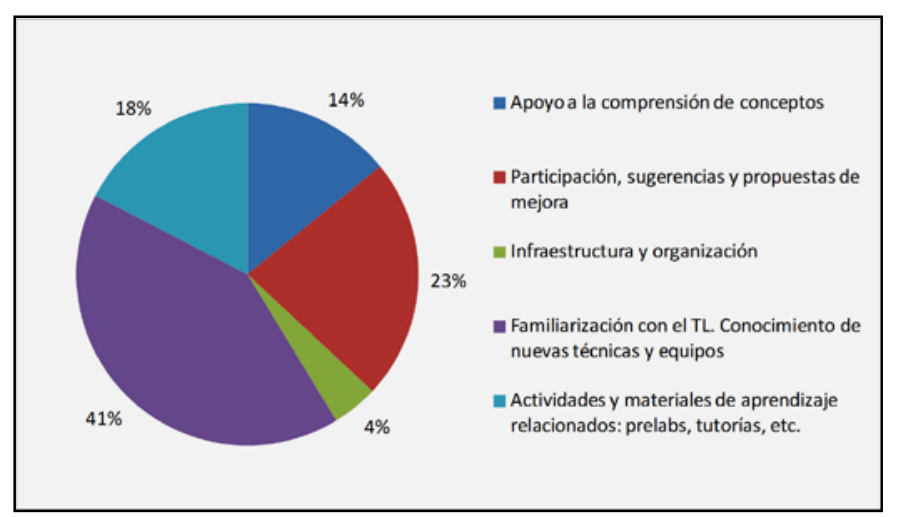

Fig. 3. Importancia relativa de los aspectos valorados positivamente en el ámbito académico

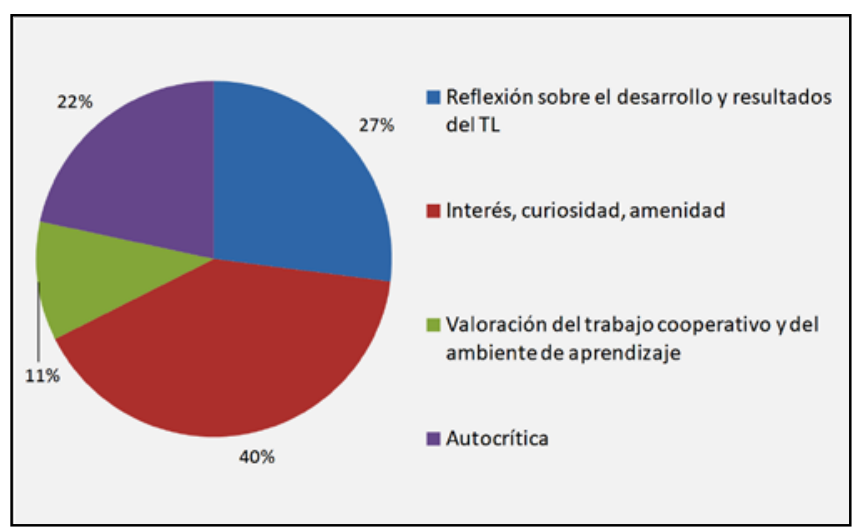

Fig. 4. Importancia relativa de los diferentes aspectos valorados positivamente en el ámbito personal y de reflexión sobre el propio aprendizaje

A partir de estos resultados cabe comentar las siguientes evidencias: en primer lugar, la conexión entre el contenido de las prácticas o aspectos relacionados con aplicaciones industriales constituye una indudable fuente de motivación. En este sentido puede afirmarse que un adecuado enfoque del trabajo experimental puede contribuir en buena medida al desarrollo de la dimensión competencial "conocimiento de los problemas contemporáneos”..

Pese a que muchos comentarios aluden a la motivación de un modo genérico, con términos como "interesante”, “amenidad”, “curiosidad”, etc., el conocimiento de nuevas técnicas y equipos muestra también ser también una importante fuente de motivación. De este modo, la dimensión competencial “instrumental específica” puede ser también objeto de especial 
atención en el TL. Ello implicaría, por ejemplo, que los estudiantes tuvieran algún tipo de contacto, aunque fuera exclusivamente a modo de demostración, con equipos y técnicas más avanzados cuyo uso guardara cierta relación con el contenido de la práctica.

Aunque no de modo mayoritario, pero sí significativo, es relevante la presencia en los informes de reflexiones, en ocasiones autocríticas, acerca del propio trabajo. El estímulo de este tipo de actividades, solicitándolas explícitamente, podría contribuir a la puesta en práctica de dimensiones competenciales tales como "análisis y resolución de problemas” y "pensamiento crítico".

La valoración de los materiales didácticos, concretamente de las actividades prelaboratorio, así como el reconocimiento de la contribución de las prácticas a la comprensión de conceptos, muestran que el estudiante puede ser más consciente de lo que a veces parece a la necesidad de integrar las prácticas en el contexto teórico de la asignatura.

Por último, es muy destacable la formulación de propuestas y sugerencias relativas al modo de organizar las prácticas. Una importante consecuencia puede ser la conveniencia de estimular la participación de los estudiantes en esta tarea. Tal vez, en principio, puede ser suficiente con pedir que a través de los informes sugieran modos alternativos concretos de gestionar el desarrollo de las sesiones, sobre todo en aquellas en que la necesidad de compartir materiales y equipos hace más difícil su organización. El plantear explícitamente que formulen tales sugerencias puede relacionarse con la dimensión competencial "planificación y gestión del tiempo".

Si se compara la información obtenida a partir de los informes con la procedente del análisis de las preguntas formuladas espontáneamente por los estudiantes durante las sesiones (Llorens-Molina et al. 2009, 2012a, 2012b) puede observarse que el análisis de éstas últimas es más útil de cara a la evaluación del grado de autonomía del estudiante y su comprensión de los objetivos del TL, aspectos claramente vinculados a las dimensiones competenciales "análisis y resolución de problemas” y "pensamiento crítico". El carácter complementario de estos dos instrumentos muestra como la triangulación en cualquier actividad de evaluación es un factor que le confiere consistencia, favoreciendo una retroalimentación efectiva.

Los principales obstáculos que los estudiantes reconocen en el desarrollo de los TL están principalmente relacionados con aspectos de infraestructura y organización, así como con los materiales docentes y su coherencia con el desarrollo real de las prácticas. (Fig. 5 y 6). En este aspecto existe una notable coincidencia con los resultados de trabajos anteriores basados en el análisis de las SGQs, a los que se hizo referencia anteriormente. 
Los informes de laboratorio. Su contribución al desarrollo y evaluación de las competencias transversales
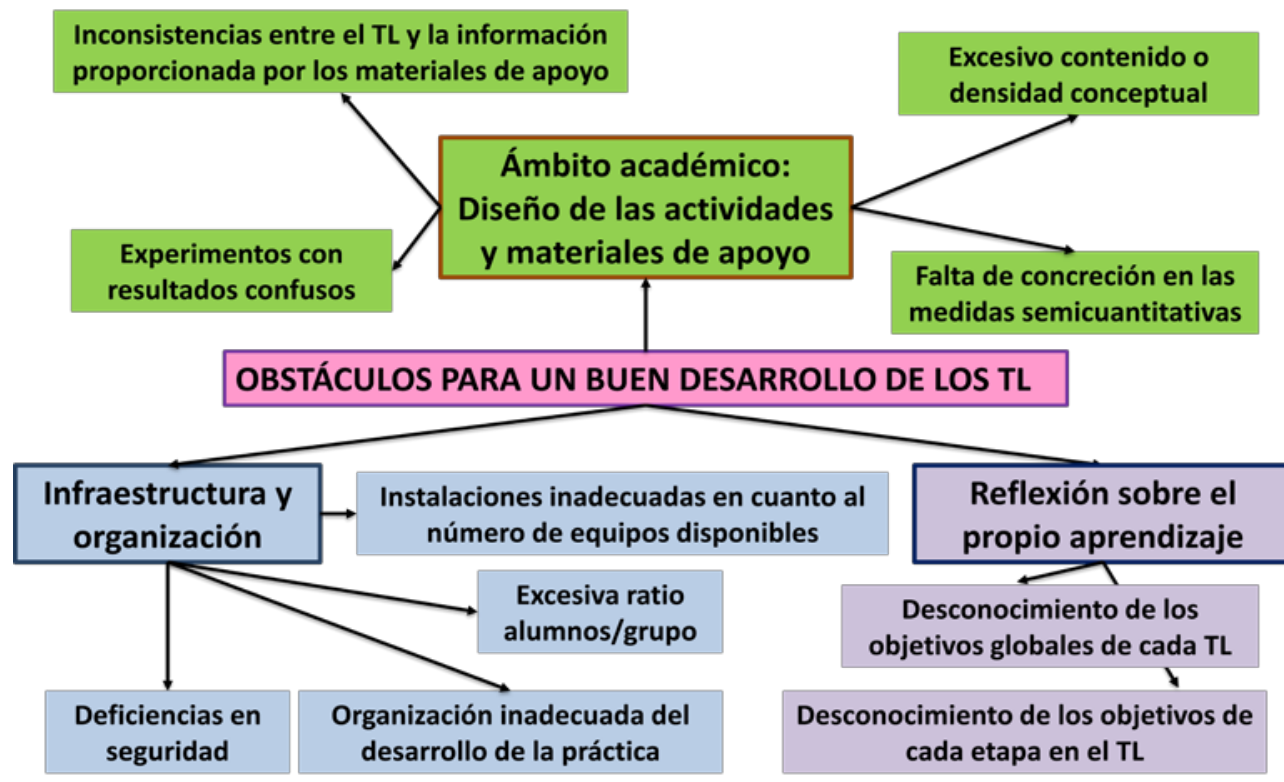

Fig. 5. Obstáculos identificados por los estudiantes en sus informes de laboratorio.

En la figura 6 se muestra la distribución cuantitativa de las citas procesadas en los tres ámbitos generales dentro del esquema de categorías elaborado.

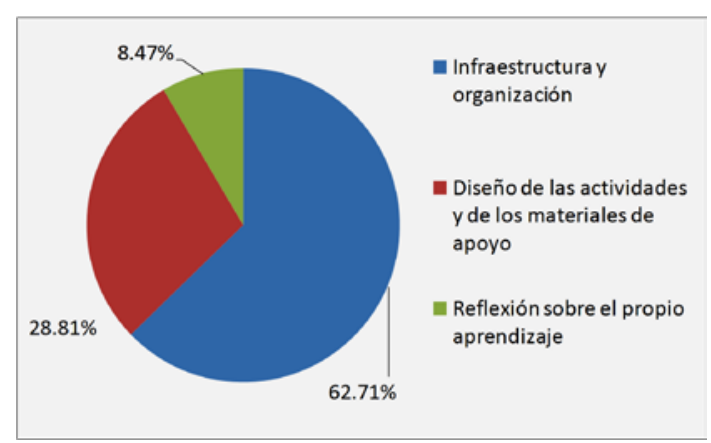

Fig. 6. Distribución cuantitativa de los obstáculos al buen desarrollo de los TL identificados por los estudiantes a través de sus informes

\section{Conclusiones}

A partir de los resultados discutidos y del análisis de la relación entre las dimensiones competenciales de la UPV y los objetivos del TL (tabla 2) es evidente que la concepción de las prácticas de laboratorio como una actividad paralela a las clases de teoría, con horarios rígidos y basada en la aplicación de una secuencia cerrada de instrucciones para verificar conocimientos preestablecidos es una visión claramente insuficiente dentro del panorama 
actual de la Educación Superior. La integración del trabajo experimental en el proceso de aprendizaje y la progresiva incorporación de actividades más abiertas y participativas son condiciones indispensables para que el trabajo de laboratorio contribuya eficazmente a la adquisición de competencias transversales y, consecuentemente, a su evaluación.

Partiendo pues de este objetivo y teniendo en cuenta la realidad actual, pueden proponerse un conjunto de buenas prácticas que contribuyan a avanzar en este sentido. En primer lugar es evidente que existen aspectos de organización e infraestructura a los que habitualmente se concede escasa atención por parecer obvios, pero que no lo son tanto y condicionan decisivamente el trabajo en el laboratorio. La atención a este tipo de cuestiones debe considerarse un prerrequisito indispensable para un trabajo experimental eficaz, antes de cualquier otra consideración. Es imprescindible, por ejemplo, planificar el uso de los equipos e instalaciones de uso colectivo (rotavapor, determinación de temperaturas de fusión, vitrinas) para mejorar la fluidez y seguridad en el trabajo. El etiquetado y distribución de los reactivos debe hacerse en absoluta concordancia con los protocolos experimentales que los estudiantes manejen y, por supuesto, al comienzo de cada curso deben analizarse cuidadosamente dichos protocolos para que recojan las modificaciones introducidas en reactivos, cantidades de los mismos, material de vidrio utilizado, etc.

La buena acogida que generalmente tiene entre los estudiantes la propuesta de actividades pre-laboratorio debe rentabilizarse introduciendo nuevos formatos y métodos, tales como la elaboración de diagramas de flujo y distribución de tareas para la planificación del trabajo (valiosa contribución a las dimensiones competenciales "aplicación pensamiento práctico", "trabajo en equipo y liderazgo" y "planificación y gestión del tiempo"). Un importante objetivo con los actuales horarios sería poder prescindir de la presentación inicial de la práctica en la sesión, liberando así tiempo para una necesaria e imprescindible puesta en común y discusión de resultados al final de la sesión.

Sería importante también, y ello es una consecuencia directa de los resultados de este estudio, que cada práctica quedara enmarcada en el contexto de una situación problemática relevante, relacionada con la vida cotidiana o con el perfil profesional de la titulación. Dicho problema, que serviría como eje motivador e hilo conductor de la práctica, debería surgir y ser discutido en las clases de teoría para avanzar en la progresiva integración de los distintos elementos de la asignatura.

En cuanto a la evaluación de los informes, es evidente también la conveniencia de incorporar actividades específicas para promover la reflexión sobre dudas, etc. Por otra parte, la utilización del "espacio compartido" de PoliformaT puede ser útil a la hora de realizar una primera revisión cuyo objetivo sería proporcionar la mínima retroalimentación para reorientar el trabajo cuando hay errores relevantes, permitiendo así su reelaboración y presentación definitiva.

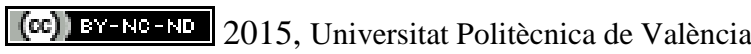

Congreso In-Red (2015) 
Los informes de laboratorio. Su contribución al desarrollo y evaluación de las competencias transversales

Por último, y a modo de conclusión general, insistir una vez más en la importancia de evaluar el trabajo de laboratorio desde el concepto de ambiente de aprendizaje. Ello requiere profundizar en un cambio en el que afortundamente podemos considerarnos inmersos: la visión sistémica de los procesos de enseñanza-aprendizaje, en la que la evaluación cobra su verdadero sentido cuando se aplica a todos los elementos que intervienen: estudiantes, profesorado y la propia institución, en sus diferentes niveles de competencia y responsabilidad.

\section{Referencias}

BROWN, K., COLE, M., (2000) "Socially Shared Cognition: System Design and the Organization of Collaborative Research”, en Jonassen, D.; Land, S. M. Eds., Theoretical Foundations of Learning Environments. Mahwah, New Jersey, US. Lawrence Erlbaum Associates, publishers, p. 197-214.

BYRNE, M.S. (1990). “More effective practical work” en Education in Chemistry, 27, p.12-13.

DUARTE, J. (2009). “Ambientes de aprendizaje: una aproximación conceptua”l en Revista Iberoamericana de Educación, www.rieoei.org/deloslectores/524Duarte.PDF (Consulta: 16 de mayo de 2015)

FISHER, D.; HARRISON, A.; HENDERSON, D., (1998) “Laboratory Learning Environments and Practical Tasks in Senior Secondary Science Classes” en Research in Science Education, 28(3), p. 353-363.

HODSON, D. (1994). “Hacia un enfoque más crítico del trabajo de laboratorio” en Enseñanza de las Ciencias. 12 (3), p. 299-313.

HOFSTEIN, A. (1996). “The Learning Environment of High Scool Students in Chemistry and Biology Laboratories” en Research in Science and Technological Education, 14 (1), p. 103-116

HOFSTEIN, A. (2004). "The laboratory in Chemistry Education: thirty years of experience with developments, implementation and research”. Chemistry Education: Research and Practice. 5(3), p. 247-264.

HOFSTEIN, A., MAMLOK-NAAMAN, R. (2007). "The laboratory in science education: the state of the art” en Chemistry education research and practice, 8(2), p. 105-107.

HOFSTEIN, A.; LUNETTA, V. N. (2003). “ The laboratory in science education: Foundations for the twenty-first century” en Science Education. 88, p. 28-54.

HOFSTEIN, A.; NAHUM, T. L.; SHORE, R., (2001) “Assessment of the Learning Environment of Inquiry-Type Laboratories in High School Chemistry” en Learning Environments Research, 4, p. 193-207. 
LLORENS-MOLINA, J. A. (2009). "Design and assessment of an online prelab model in general chemistry: A case study”. Journal of the Research Center for Educational Technology, 4(2), p. 15-31.

LLORENS-MOLINA, J. A., DE JAIME, J. M. L., BERZOSA, I. S. (2012). “Analysis of students' generated questions in laboratory learning environments”. Journal of Technology and Science Education, 2(1), p. 46-55.

LLORENS-MOLINA, J. A., SANZ BERZOSA, I., LLORENS DE JAIME, J. M. (2012). “La caracterización del ambiente de aprendizaje en un laboratorio de química general mediante métodos de investigación social” en Enseñanza de las ciencias, 30, p. 5 -22.

McROBBIE, C. J.; GIDDINGS, G. J.; FRASER, B. J. (1990). "Research into the Environment of Science Laboratory Classes in Australian Schools” en Research in Science Education, 20, p. 200-209. PEDROSA DE JESUS, H.; ALMEIDA, P. TEIXEIRA-DIAS, J. J., WATTS, M. (2007). "Where learner's questions meet modes of teaching: A study of cases”. Research in Education, 78(1), p. 1-20.

PEDROSA DE JESUS, H.; TEIXEIRA-DIAS, J. J., WATTS, M. (2003). “Questions of Chemistry” en International Journal of Science Education, 25(8), p. 1015-1034.

VILLA, A., POBLETE, M. (2011) “Evaluación de competencias genéricas: principios, oportunidades y limitaciones” en Bordon, 63 (1), p. 147-170. 